\title{
Awareness Regarding Vitamin D Amongst The Medical And Non-medical Students Of Karachi
}

Asma Dahar, Tahira Assad, Hafiza Touseef Sayyar

\section{- - - - - - - - - - - - - - - - - - - - - - - - - - - - - - - - \\ ABSTRACT}

Objective: To assess the awareness regarding Vitamin D amongst medical and non-medical students.

Study Design and Setting: A cross-sectional study was conducted during the period December 2016-June 2017 on a sample size of 388 subjects.

Methodology: Undergraduate medical and dental students that are in their 3rd or 4th year, house officers, post graduate trainees and graduate medical students were included in the study. Undergraduate and graduate students from non-medical institutes were also included. The participants were assessed on the basis of a questionnaire which contained 15 closed ended questions.

Results: The age of participants in this research varied from 21 to 35 years with the mean age of 24. Male subjects were $25 \%$ but the females were predominant in this study, calculating $75 \%$. Medical subjects were $70.4 \%(\mathrm{n}=273)$ and Non-

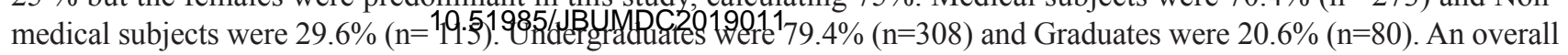
of $75 \%$ of the medical subjects have satisfactory knowledge as compared to $25 \%$ of the non-medical subjects.

Conclusion: There is a lack of awareness regarding vitamin D among non-medical students. Awareness of vitamin among medical students was also not up to the mark.

Keywords: Awareness, medical and non-medical students and Vitamin D,

\section{INTRODUCTION:}

Medical and allied health professionals are the first referrals to whom the patients present, when they have signs of vitamin D deficiency/insufficiency. Due to cosmopolitan life style, our local population is not exposed to sufficient amount of sunlight that is required for vitamin D activation. Latest literature shows that, Vitamin D which is also known as the sunshine vitamin, plays a vital role in fighting against cancer, heart diseases, bone fractures, type 2 diabetes, depression, influenza and autoimmune diseases. ${ }^{1}$ This vitamin is not solely consumed through diet but it is produced endogenously by the action of sunlight on skin as well. ${ }^{2}$ Vitamin D is classified into two types: Vitamin D2 and Vitamin D3. The Former is also known as Ergocalciferol while the latter is also known as Cholecalciferol. There are two sources of Vitamin D3. First, being the exogenous source that is obtained from diet and the second is the endogenous source, in which vitamin D3 is synthesized

\begin{tabular}{|c|}
\hline $\begin{array}{l}\text { Asma Dahar } \\
\text { Dental Student } \\
\text { Fatima Jinnah Dental College } \\
\text { Azam town, Karachi. }\end{array}$ \\
\hline $\begin{array}{l}\text { Tahira Assad } \\
\text { Associate Professor } \\
\text { Karachi Institute of Medical sciences } \\
\text { Malir Cantt, Karachi. } \\
\text { Email:drtahiraassad@gmail.com }\end{array}$ \\
\hline $\begin{array}{l}\text { Hafiza Touseef Sayyar } \\
\text { Pharmacist } \\
\text { Bahria University Medical and Dental College, } \\
\text { Karachi. }\end{array}$ \\
\hline $\begin{array}{l}\text { Received: 01-02-2019 } \\
\text { Accepted: 14-05-2019 }\end{array}$ \\
\hline
\end{tabular}

through sunlight. Regardless the source from which the Vitamin D3 comes, it has to undergo two hydroxylation processes. The first hydroxylation process occurs in the liver, where it forms 25-hydroxy vitamin D3 [25(OH) D], and then second hydroxylation process occurs in the kidney to form 1, 25 hydroxy vitamin D3 (activated form). Vitamin D2 has lower binding capacity to proteins and has a rapid clearance, whereas Vitamin D3 has a half-life of around 23 weeks, this is the reason behind more potency of Vitamin D3 than Vitamin D2. ${ }^{3}$

It is seen that deficiency of vitamin $\mathrm{D}$ affects over one billion people across the globe as it is the most under diagnosed and untreated of all the deficiencies. ${ }^{4}$ Defects in mineralization of the skeletal components of our body are caused by Vitamin D deficiency. These mineralization deficits provide little anatomical support for the periosteal covering. Due to these deficits, patients suffering from osteomalacia complain of generalized arthralgia and myalgia. Patients with mineralization defects of bones are often misdiagnosed with fibromyalgia, dysthymia, degenerative joint disease, arthritis, chronic fatigue syndrome and other diseases. ${ }^{5-6}$

The prevalence of vitamin D deficiency has been found all over the world except for a few areas in South and Southeast Asia. ${ }^{7}$ Studies have shown that rickets is common in all age groups in Pakistan, Bangladesh and India. ${ }^{8}$ Mothers with deficiency of Vitamin D and low intake of calcium in their diet were found to be the cause of rickets in Pakistan, Bangladesh and India. ${ }^{9}$ A survey conducted in 2007 by International Osteoporosis Foundation showed that people are familiar with the role that calcium plays as a bone constructing agent but are not apprehensive about the role 
of Vitamin D in preservation of a healthy body. ${ }^{10}$ In a study done in 2007, Mc Gillivray and his colleagues realised that out of a total of 232 East African migrant children and adolescents aged 0-17 years who were residing in Melbourne, the prevalence of vitamin D deficiency (serum 25-OHD level $<25 \mathrm{nmol} / \mathrm{l}$ ) was $44 \%$ and that of vitamin D insufficiency (serum $25-\mathrm{OHD}$ level $<50 \mathrm{nmol} / \mathrm{l}$ ) was $87 \%$. Risk factors for vitamin D deficiency in these subjects included children less than 5 years of age, female, prolonged stay in Australia, reduced sunlight exposure and sping or winter season. ${ }^{11}$ According to a report, maximal risk of Vitamin D insufficiency and decreased calcium absorption is seen in young adults aged between $20-39$ years. ${ }^{12}$ In a study conducted in Karachi, it was observed that high prevalence of vitamin D deficiency was found in females. The prime predictors for the vitamin D deficiency were found to be Age, location of residence and housing structure. 13

In Pakistan, basic needs of people are not fulfilled and the healthcare systems are not up to the mark. Health education and health care systems do not meet the criteria of public's health demand. ${ }^{14}$ It is due to this breach in health education system that we are facing outcomes in which there are high prevalence of vitamin D deficiency.

The objective of this study was to assess the awareness of Vitamin D amongst medical and non-medical students. It is very influential to assess the awareness levels among medical and non-medical groups as this will predict the future health issues.

\section{METHODOLOGY:}

A descriptive cross-sectional study was conducted during the period December 2016-June 2017. Ethical review board of Fatima Jinnah Dental College and Hospital provided Ethical approval, with code DEC-2016-ORS-01 to conduct this study.

Undergraduate medical and dental students that are in their 3rd or 4th year, house officers, post graduate trainees and graduate medical professionals were included in the study. Undergraduate and graduate students from non-medical institutes were also included. A self-explanatory pre-tested questionnaire was given in various medical, dental and nonmedical schools which contained 15 close ended questions. ${ }^{15}$

The sampling technique chosen was non probability convenient Sampling technique. The sample size was calculated using sample size calculator on www.openepi.com at $95 \%$ confidence level and prevalence of $50 \%$. The sample size used for this study was 388 which excluded Nonpracticing dental professionals; Post graduate non-medical students and Practising medical doctors.

Prior consent was obtained from every respondent before including in the study. A data entry sheet was formulated and filled by the participants that had questions regarding their age, gender, education and level of education. Data tabulation and analysis was completed using SPSS software version 22. A Chi -square test was used to determine awareness among them.

\section{RESULTS:}

Data was entered in IBM SPSS statistics version 22. Percentages, standard deviation, Odds ratio and Chi square tests were applied to all the Qualitative questions. The total subjects who participated in this research were 388 .

\section{General Characteristics}

The age of participants in this research varied from 21 to 35 years with the mean age of 24 . Male subjects were $25 \%$ but the females were predominant in this study, calculating $75 \%$. Medical subjects were $70.4 \%(n=273)$ and Nonmedical subjects were $29.6 \%(n=115)$. Undergraduates were $79.4 \%(n=308)$ and Graduates were 20.6\% $(n=80)$. (Table 1), Table 2 shows the percentage of medical and non- medical students answered positively on various questions asked regarding vitamin $\mathrm{D}$. (Table 2 ), Table 3 shows statistically the comparison between medical and non- medical professionals regarding their awareness of vitamin D. Although medical professionals were found to be more knowledgeable than non-medical professionals as expected, but their knowledge was also not upto the mark.

\section{DISCUSSION:}

Vitamin D deficiency is common all over the world but it is more prevalent in Pakistan ${ }^{16}$. The main function of vitamin $\mathrm{D}$ is to maintain the balance of calcium absorption and excretion in the body ${ }^{17}$. A study was conducted in Manchester, UK to check the awareness of vitamin D among people at risk of vitamin D. A total of 363 people were included in the study whose ages were above 18 years. In this study, one hundred and sixty people $(72 \%)$ had heard about vitamin $\mathrm{D}$ and 61 of the respondents $(28 \%)$ had never heard of vitamin D. ${ }^{18}$ Another study was conducted in New Delhi, in which 599 students aged between 18 to 25 years were included. Amongst them, 99.7\% females answered that they heard about vitamin D before and $99 \%$ of the male answered the same. ${ }^{19}$

In this study, $76.5 \%$ of the medical population answered correctly about the production of vitamin D. Moreover,

Table 1: Gender, Category and level of education wise distribution of the subjects

\begin{tabular}{|l|l|c|}
\hline \multicolumn{1}{|c|}{ Group } & Item & Percentage \\
\hline \multirow{2}{*}{ Gender } & Male & 25.0 \\
\cline { 2 - 3 } & Female & 75.0 \\
\hline \multirow{2}{*}{ Category } & Medical & 70.4 \\
\cline { 2 - 3 } & Non-medical & 29.6 \\
\hline \multirow{2}{*}{ Level of Education } & Under-graduate & 79.4 \\
\cline { 2 - 3 } & Graduate & 20.6 \\
\hline
\end{tabular}


Asma Dahar, Tahira Assad, Hafiza Touseef Sayyar

Table 2: Percentage of medical and non-medical students answered positively on various questions asked

\begin{tabular}{|l|l|l|l|}
\hline \multicolumn{1}{|c|}{ Questions } & Medical (\%) & Non-medical (\%) & Total (\%) \\
\hline Have you ever heard of Vitamin D? & $70.9 \%(271 / 382)$ & $29.1 \%(111 / 382)$ & $98.5 \%(382 / 388)$ \\
\hline Is Vitamin D produced inside the body by itself? & $76.5 \%(153 / 200)$ & $23.5 \%(47 / 200)$ & $51.5 \%(200 / 388)$ \\
\hline Is Vitamin D good for bone health? & $72.5 \%(272 / 375)$ & $27.5 \%(103 / 375)$ & $96.6 \%(375 / 388)$ \\
\hline Do you think your Vitamin D status is sufficient? & $70.1 \%(108 / 154)$ & $29.9 \%(46 / 154)$ & $39.7 \%(154 / 388)$ \\
\hline Have you ever got your Vitamin D levels checked? & $69.9 \%(100 / 143)$ & $30.1 \%(43 / 143)$ & $36.9 \%(143 / 388)$ \\
\hline Is Vitamin D produced in the body with the help of sunlight? & $70.9 \%(254 / 350)$ & $27.4 \%(96 / 350)$ & $90.2 \%(350 / 388)$ \\
\hline Do you take sun protection while any outdoor activity? & $70.6 \%(115 / 163)$ & $29.4 \%(48 / 163)$ & $42.0 \%(163 / 388)$ \\
\hline What are the normal levels of vitamin D in serum & $72.2 \%(39 / 54)$ & $27.7 \%(15 / 54)$ & $13.91 \%(54 / 388)$ \\
\hline What should be the minimum daily intake of Vitamin D? & $74.6 \%(44 / 59)$ & $25.4 \%(15 / 59)$ & $15.2 \%(59 / 388)$ \\
\hline $\begin{array}{l}\text { What is the average time needed to spend in sunlight to have } \\
\text { enough vitamin D? }\end{array}$ & $72.2 \%(172 / 238)$ & $27.7 \%(66 / 238)$ & $61.3 \%(238 / 388)$ \\
\hline
\end{tabular}

Table 3: Comparison between medical and non-medical students based on certain questions inquired regarding vitamin D

\begin{tabular}{|c|c|c|c|c|c|c|}
\hline Question Asked & Yes & No & Don't know & Odds Ratio & $95 \%$ CI. & P-Value \\
\hline \multicolumn{4}{|c|}{ 1. Have you heard of Vitamin D? } & \multirow{3}{*}{4.883} & \multirow{3}{*}{$0.882-27.043$} & \multirow{3}{*}{0.066} \\
\hline Medical & 271 & 2 & 0 & & & \\
\hline Non-Medical & 111 & 4 & 0 & & & \\
\hline \multicolumn{4}{|c|}{ 2. Is Vitamin D produced in the body by itself? } & \multirow{3}{*}{1.502} & \multirow{3}{*}{$0.936-2.411$} & \multirow{3}{*}{0.058} \\
\hline Medical & 153 & 104 & 16 & & & \\
\hline Non-Medical & 47 & 48 & 20 & & & \\
\hline \multicolumn{4}{|c|}{ 3. Is Vitamin D good for bone health? } & \multirow{3}{*}{7.922} & \multirow{3}{*}{$0.815-77.029$} & \multirow{3}{*}{0.068} \\
\hline Medical & 272 & 1 & 0 & & & \\
\hline Non-Medical & 103 & 3 & 9 & & & \\
\hline \multicolumn{4}{|c|}{ 4. Do you think your Vitamin D status is sufficient? } & \multirow{3}{*}{0.875} & \multirow{3}{*}{$0.532-1.439$} & \multirow{3}{*}{0.345} \\
\hline Medical & 106 & 104 & 47 & & & \\
\hline Non-Medical & 39 & 36 & 20 & & & \\
\hline \multicolumn{4}{|c|}{ 5. Have you ever got your Vitamin D checked? } & \multirow{3}{*}{0.849} & \multirow{3}{*}{$0.535-1.349$} & \multirow{3}{*}{0.282} \\
\hline Medical & 92 & 159 & 6 & & & \\
\hline Non-Medical & 40 & 46 & 9 & & & \\
\hline \multicolumn{4}{|c|}{ 6. Is Vitamin D Produced in the body with the help of sunlight? } & \multirow{3}{*}{2.165} & \multirow{3}{*}{$0.870-5.387$} & \multirow{3}{*}{0.079} \\
\hline Medical & 241 & 11 & 5 & & & \\
\hline Non-Medical & 84 & 6 & 5 & & & \\
\hline \multicolumn{4}{|c|}{ 7. Do you take sun protection while any outdoor activity } & \multirow{3}{*}{0.939} & \multirow{3}{*}{$0.579-1.518$} & \multirow{3}{*}{0.445} \\
\hline Medical & 109 & 108 & 40 & & & \\
\hline Non-Medical & 41 & 39 & 15 & & & \\
\hline
\end{tabular}

$70.1 \%$ of the medical subjects and $29.9 \%$ of the non-medical subjects thought that their Vitamin D levels are sufficient.

Keeping the levels of all the minerals and vitamins adequate is crucial for a healthy body. Vitamin D being an essential vitamin needs to be within normal levels in our blood as prescribed (54-90ng/mL for sunny countries). ${ }^{20}$ Vitamin D is needed for maintaining the levels of calcium and phosphate in our blood. It promotes absorption of Calcium and phosphorus in our blood. It also causes normal bone mineralization and prevents tetany caused by hypocalcaemia. ${ }^{21}$ Additionally, vitamin D has some noncalcemic effects including its effects on cell growth, body's natural defence system, neuromuscular functions, carcinogenesis and its cardiovascular functions. ${ }^{22}$

In this study, a cumulative of $36.9 \%$ students answered "Yes" on inquiring about assessment of their vitamin D levels. Among them 69.9\% were medical students and 30.1\% were non-medical. Periodic assessment of vitamin D levels 
is paramount in prevention of disorders of bone, nutritional problems, organ malfunction and many other medical conditions as well. Sufficient levels of Vitamin D prevent many diseases like rickets in children, osteomalacia and osteoporosis in adults. ${ }^{21}$

A cumulative of $90.2 \%$ answered (Yes) when asked about the role of sunlight in the production of vitamin $\mathrm{D}$ which is in accordance to other studies. ${ }^{23-25}$ Vitamin D formation is dependent on a sufficient amount of sun exposure but according to a number of studies, sun exposure is a big problem in most of the countries as UV radiation is a carcinogen and can cause skin cancer. ${ }^{22,28}$

Results from a study suggest that less enthusiasm to sun's exposure was linked to a person's Vitamin D knowledge, female gender and if she had higher education. In this study, $62.3 \%$ of the people did not prefer going into the sun and many others took sun protection to avoid their sun exposure. These people were mostly young Chinese women who had enough Vitamin D knowledge and awareness. ${ }^{23}$

$72.2 \%$ of the medical respondents and $27.2 \%$ of the nonmedical respondents replied 30 minutes per day as the average time needed to spend in sunlight, which is the correct answer. As it has been suggested by some vitamin D researchers, people should get 5 to 30 minutes of sun exposure between $10 \mathrm{Am}$ to $3 \mathrm{AM}^{29}$

In another study done on athletes in New Zealand, $76 \%$ of Athletes had great information on the role of sun as a source of vitamin D. ${ }^{24}$ Awareness on the topic of sun exposure and how much sunlight a person needs to obtain for activation of Vitamin D should be given. Brand et al. found in his study that the participants who were Australian women were highly aware of this. ${ }^{25}$

In this study, $72.2 \%$ medical subjects and $27.7 \%$ non-medical subjects answered correctly regarding normal levels of vitamin $\mathrm{D}$, stating that greater than or equal to $50 \mathrm{nmol} / \mathrm{L}$ is the normal level for vitamin $\mathrm{D}$ in serum. As recorded from a report by National Institute of health published in the year 2018 , people with serum levels of vitamin D less than $30 \mathrm{nmol} / \mathrm{L}$ are suffering from vitamin $\mathrm{D}$ deficiency; serum levels of $30-50 \mathrm{nmol} / \mathrm{L}$ are considered less for good bone and overall health but levels of greater than $50 \mathrm{nmol} / \mathrm{L}$ are regarded are adequate for bone and overall health in healthy people. Levels greater than $125 \mathrm{nmol} / \mathrm{L}$ are accompanied with potential adverse effects. ${ }^{26}$ In a study conducted in Karachi, it was seen that many people who were apparently healthy also faced vitamin D deficiency and insufficiency. These individuals were seen to be at risk for developing musculoskeletal and other chronic diseases. It was concluded in this study that serum parathyroid hormone levels and serum 25 OHD levels are considered better markers for vitamin D deficiency and insufficiency. ${ }^{27}$

In this study, $74.6 \%$ of medical subjects and $25.4 \%$ nonmedical subjects said that $600 \mathrm{IU}$ per day is the average daily intake of vitamin D. According to the report, $600 \mathrm{IU}$ per day is the adequate daily intake of vitamin $\mathrm{D}$ for a healthy individual aged 1 to 70 years. ${ }^{28}$

The sample size of medical and non-medical population was not same in this study. More researches and awareness on the topic of "Vitamin D" should be promoted.

\section{CONCLUSION:}

The results of this study interpret that there is a lack of awareness regarding vitamin $\mathrm{D}$ among non-medical students. Although Medical students were found to be more knowledgeable than non- medical students as expected, their knowledge was also not up-to the mark.

\section{REFERENCES}

1. Nair R, Maseeh A. Vitamin D: The "sunshine" vitamin. Journal of pharmacology \& pharmacotherapeutics. 2012; 3(2):118.

2. Major JM, Kiruthu C, Weinstein SJ, Horst RL, Snyder K, Virtamo J, Albanes D. Pre-diagnostic circulating vitamin D and risk of melanoma in men. PLoS One. 2012;7(4):e35112.

3. Malaeb D, Hallit S, Salameh P. Assessment of vitamin D levels, awareness among Lebanese pharmacy students, and impact of pharmacist counseling. Journal of epidemiology and global health. 2017;7(1):55-62.

4. Riaz H, Finlayson A, Bashir S, Hussain S, Mahmood S, Malik F, et al. Prevalence of Vitamin D deficiency in Pakistan and implications for the future. Expert Review of Clinical Pharmacology. 2016;9(2):329-38.

5. Plotnikoff GA, Quigley JM. Prevalence of severe hypovitaminosis D in patients with persistent, nonspecific musculoskeletal pain. InMayo clinic proceedings 2003; 78(12): 1463-70.

6. Holick MF, Chen TC. Vitamin D deficiency: a worldwide problem with health consequences-. The American journal of clinical nutrition. 2008;87(4):1080S-6S.

7. Mithal A, Wahl DA, Bonjour JP, Burckhardt P, DawsonHughes B, Eisman JA, Fuleihan GE, Josse RG, Lips P, MoralesTorres J, IOF Committee of Scientific Advisors (CSA) Nutrition Working Group. Global vitamin D status and determinants of hypovitaminosis D. Osteoporosis international. 2009;20(11):1807-20.

8. Pai B, Shaw N. Understanding rickets. Paediatrics and Child Health. 2011;21(7):315-321.

9. Pettifor JM. Vitamin D \&/or calcium deficiency rickets in infants \& children: a global perspective. Indian Journal of Medical Research. 2008;127(3):245-249.

10. Chan SP, Scott BB, Sen SS. An Asian viewpoint on the use of vitamin D and calcium in osteoporosis treatment: physician and patient attitudes and beliefs. BMC musculoskeletal disorders. 2010;11(1):248.

11. McGillivray G, Skull SA, et.al. High prevalence of asymptomatic vitamin-D and iron deficiency in East African immigrant children and adolescents living in a temperate climate. Archives of Disease in Childhood. 2007 Sep 3.

12. Whiting SJ, Langlois KA, Vatanparast H, Greene-Finestone LS. The vitamin D status of Canadians relative to the 2011 Dietary Reference Intakes: an examination in children and adults with and without supplement use-. The American journal of clinical nutrition. 2011;94(1):128-35. 
13. Khan AH, Iqbal R, Naureen G, Dar FJ, Ahmed FN. Prevalence of vitamin $\mathrm{D}$ deficiency and its correlates: results of a community-based study conducted in Karachi, Pakistan. Archives of Osteoporosis. 2012;7(1-2):275-82.

14. Anjum F, GhayasS, Rizvi N : awareness and knowledge of calcium and vitamin D among pharmacy students in karachi,pakistan : Journal of hospital pharmacy 2014; 9(3): 3.

15. Arora H, Dixit V, Srivastava N. Evaluation of knowledge, practices of vitamin $\mathrm{d}$ and attitude toward sunlight among indian students. Asian Journal of Pharmaceutical and Clinical Research. 2016;9(1).

16. Anjum P, Safder N, Khalid M, Mehboob I. Vitamin D deficiency in Pakistani population. Journal of Pakistan Orthopaedic Association. 2013;25(1):18-9.

17. Lips P. Vitamin D physiology. Progress in Biophysics and Molecular Biology. 2006;92(1):4-8.

18. Alemu E, Varnam R. Awareness of vitamin D deficiency among at-risk patients. BMC research notes. 2012;5(1):17.

19. Alshahrani F, Aljohani N. Vitamin D: deficiency, sufficiency and toxicity. Nutrients. 2013;5(9):3605-16.

20. Del Valle HB, Yaktine AL, Taylor CL, Ross AC, editors. Dietary reference intakes for calcium and vitamin D. National Academies Press; 2011 Apr 30.

21. Samuel S, Sitrin MD. Vitamin D's role in cell proliferation and differentiation. Nutrition reviews. 2008;66:S116-24
22. Ho-Pham L, Nguyen M. Survey on knowledge and attitudes on Vitamin D and sunlight exposure in an urban population in Vietnam. Journal of the ASEAN Federation of Endocrine Societies. 2014;27(2):191.

23. Walker N, Love TD, Baker DF, Healey PB, Haszard J, Edwards AS, Black KE. Knowledge and attitudes to vitamin D and sun exposure in elite New Zealand athletes: a cross-sectional study. Journal of the International Society of Sports Nutrition. 2014;11(1):47.

24. Brand CA, Abi HY, Couch DE, Vindigni A, Wark JD. Vitamin D deficiency: a study of community beliefs among dark skinned and veiled people. International journal of rheumatic diseases. 2008;11(1):15-23.

25. NIH Office of Dietary Supplements. U.S. Department of Health and Human Services; [cited 2019Apr10]. Available from: https://ods.od.nih.gov/factsheets/Vitamin DHealthProfessional/

26. Mansoor S, Habib A, Ghani F, Fatmi Z, Badruddin S, Mansoor $\mathrm{S}$, Siddiqui I, Jabbar A. Prevalence and significance of vitamin D deficiency and insufficiency among apparently healthy adults. Clinical biochemistry. 2010;43(18):1431-5.

27. Wolpowitz D, Gilchrest BA. The vitamin D questions: how much do you need and how should you get it? Journal of the American Academy of Dermatology. 2006;54(2):301-17.

28. Heaney RP, Holick MF. Why the IOM recommendations for vitamin D are deficient. Journal of Bone and Mineral Research. 2011;26(3):455-7.

29. Holick MF. Vitamin D deficiency. New England Journal of Medicine. 2007;357(3):266-81. 